\title{
A New Unsteady Model for Dense Cloud Cavitation in Cryogenic Fluids
}

\author{
A. Hosangadi ${ }^{*}$ and V. Ahuja. ${ }^{\dagger}$ \\ Combustion Research and Flow Technology, Inc. (CRAFT Tech) \\ 6210 Keller's Church Road \\ Pipersville, PA 18947 USA \\ hosangad@craft-tech.com
}

\begin{abstract}
A new unsteady, cavitation model is presented wherein the phase change process (bubble growth/collapse) is coupled to the acoustic field in a cryogenic fluid. It predicts the number density and radius of bubbles in vapor clouds by tracking both the aggregate surface area and volume fraction of the cloud. Hence, formulations for the dynamics of individual bubbles (e.g. Rayleigh-Plesset equation) may be integrated within the macroscopic context of a dense vapor cloud i.e. a cloud that occupies a significant fraction of available volume and contains numerous bubbles. This formulation has been implemented within the CRUNCH CFD, which has a compressible "real" fluid formulation, a multi-element, unstructured grid framework, and has been validated extensively for liquid rocket turbopump inducers. Detailed unsteady simulations of a cavitating ogive in liquid nitrogen are presented where time-averaged mean cavity pressure and temperature depressions due to cavitation are compared with experimental data. The model also provides the spatial and temporal history of the bubble size distribution in the vapor clouds that are shed, an important physical parameter that is difficult to measure experimentally and is a significant advancement in the modeling of dense cloud cavitation.
\end{abstract}

$\mathrm{N}$ UMERICAL simulation of cavitating flows and specifically the development of phase change models has received enormous attention from researchers. Yet all the models currently used in CFD codes have major drawbacks for dense cavitating flows. Cavitation models in CFD tools may broadly be classified into two categories: a) A bubbly framework using the Rayleigh-Plesset equation, and b) Continuum formulation solving for vapor phase transport. There have been numerous numerical studies using the bubbly closure model (e.g., see Ref. $[1,2]$ ). Here, the flowfield is seeded with bubbles and the change in bubble mass (and therefore the mixture density in each cell) is obtained from the Rayleigh-Plesset equations. The advantage of this formulation is that a physical model describes the cavitation phase change. However, the key limitation of these studies arises from their implicit restriction that the mixture be "dilute" i.e., the vapor void fraction $\left(\phi_{g}\right)$ is negligibly small. For instance, Kubota et al., define mixture density as $\rho_{m}=\left(1-\phi_{g}\right) \rho_{l}$ which neglects the hydrodynamics of the vapor phase. This is a severe restriction. In particular, for cloud cavitation problems where the vapor clouds are dense and the spatial extent of each distinct cloud can be large, the hydrodynamics of the vapor phase has to be resolved numerically.

The second approach followed is a continuum formulation that is applicable to dense cavitating flow (e.g., Ref. [3-6]) among many other works in the literature). Here, a separate equation for the transport of the vapor phase is solved for and there is no restriction on the vapor volume fraction being small. The cavitation process is modeled as a phase change source term. The primary drawback of these previous works has been that the cavitation source term is specified in an ad-hoc fashion and does not account for the bubble dynamics. For instance, the barotropic model ${ }^{3}$ completely decouples the time scale of cavitation and enforces a user specified density-pressure relationship. The model by Singhal ${ }^{5}$ does allow for finite time scale with a rate equation for phase change based on the local pressure. However, the key limitation here is that the neither the radius nor the number density of the bubbles in the vapor cloud are estimated. Since the net phase change due to cavitation results from the combination of bubble number

\footnotetext{
- Principal Scientist, 6210 Keller's Church Road, Pipersville, PA 18947, and Senior Member.

† Research Scientist, 6210 Keller's Church Road, Pipersville, PA 18947, and Senior Member.
} 
density and the individual bubble radius change, these formulations are ad-hoc and not adequate for unsteady cloud cavitation.

The primary contribution of our work is the development of a fundamental model for cavitation that integrates the two approaches discussed above. We solve for the transport of vapor phase and are not restricted to dilute multiphase flows. However, in addition we track the number density and radius of the bubbles associated in the vapor cloud by solving an additional equation for the total surface area associated with a bubbly cloud. This allows us to integrate fundamental bubble dynamic equations for changes in mean bubble radius and provides an accurate model for applications with extensive cavitation. The details of the formulation are given in the sections below.

In the sections to follow, we give a brief overview of the "compressible" framework for modeling cavitating cryogenic fluids followed by a discussion of the new unsteady cavitation model developed. The series of rigorous validation tests performed to verify both the accuracy of the model to track individual bubble dynamics, as well as the larger macroscopic evolution of the vapor cloud are described next. The model has been tested on the following problems in water: 1) Unsteady bubbly pipe flow, 2) Unsteady cloud cavitation on a cavitating ogive in liquid nitrogen. As we discuss below, the results indicate that the model performed well and yielded fundamental insight into unsteady cavitation physics.

\section{Multi-Phase Equation System}

We give a very brief overview of the basic multiphase equation system here and refer the reader to Hosangadi and Ahuja ${ }^{7}$ for more details. The equation system is written solved in a pressure based form as:

$$
\begin{gathered}
\Gamma \frac{\partial Q_{v}}{\partial t}+\frac{\partial E}{\partial x}+\frac{\partial F}{\partial y}+\frac{\partial G}{\partial z}=\mathrm{S}+\mathrm{D}_{\mathrm{v}} \\
Q_{v}=\left(\begin{array}{c}
P \\
u \\
v \\
w \\
\phi_{g} \\
h_{m} \\
k \\
\varepsilon
\end{array}\right) \quad E=\left(\begin{array}{c}
\rho_{m} u \\
\rho_{m} u^{2}+P \\
\rho_{m} u v \\
\rho_{m} u w \\
\rho_{g} \phi_{g} u \\
\rho_{m} h_{m} u \\
\rho_{m} k u \\
\rho_{m} \varepsilon u
\end{array}\right) \quad S=\left(\begin{array}{c}
0 \\
0 \\
0 \\
0 \\
m_{t} \\
m_{t} h_{f g} \\
S_{k} \\
S_{\varepsilon}
\end{array}\right)
\end{gathered}
$$

The vectors $\mathrm{Qv}, \mathrm{E}$ and $\mathrm{S}$ are given above. The matrix $\Gamma\left(=\partial Q / \partial Q_{v}\right)$ defines the transformation from the conservative to primitive variables and may further be preconditioned to obtain an efficient time-marching scheme (we refer the reader to Ref. [7] for more details).

The source term for the vapor phase arises due to cavitation where $m_{t}$ is the net rate of vapor mass generation (or condensation), and the corresponding source term for the energy equation is given as $m_{t} h_{f g}$ where $h_{f g}$ is the change in enthalpy resulting from the phase change and is a function of the local fluid temperature. These phase change source terms are discussed in a later section.

The mixture density, enthalpy, and vapor porosity are related by the following relations locally in a given cell volume:

$$
\begin{gathered}
\rho_{m}=\rho_{g} \phi_{g}+\rho_{L} \phi_{L} \\
\rho_{m} h_{m}=\rho_{g} \phi_{g} h_{g}+\rho_{L} \phi_{L} h_{L} \\
1=\phi_{g}+\phi_{L}
\end{gathered}
$$

where $\rho_{\mathrm{g}}, \rho_{\mathrm{L}}$ are the physical material densities, while $h_{g}$ and $h_{L}$ are the sensible enthalpy of the vapor and liquid phase respectively, and in general are functions of both the local temperature and pressure. In our study here, these properties were generated from the Standard thermodynamic database 12 available from NIST for pure fluids ${ }^{8}$. The thermodynamic properties of the fluid where specified using the saturation values from the table corresponding to the local temperature of the fluid. Equations (1-2) represent a stiff system with large variations in the acoustic speed 
that are a function of the local multi-phase composition. Preconditioning techniques are used to overcome this stiffness and obtain an efficient numerical scheme ${ }^{7}$.

\section{Unsteady Cavitation Model Using Cloud Surface Area Equation}

The new, unsteady cavitation model developed incorporates formulations for bubble dynamics (e.g. RayleighPlesset equation) as an integral part of dense cloud cavitation. By drawing on our experience in dense spray combustion ${ }^{9}$, we track the surface area associated with the cavitation cloud as it evolves spatially and temporally. We note that this Eulerian procedure has been formulated within the premise of a dense bubble cloud where a large number of bubbles are present. Bubbles comprising the cloud will be characterized by their Sauter mean radius, which preserves the ratio of the surface area and volume for the bubbly cloud. Thus, the source term for cavitation will have two independent factors controlling it: a) the net surface area given by the bubble Sauter mean radius, and b) the rate of change of the radius, which may be specified using the Rayleigh-Plesset equation.

The additional equation (shown in 1-D form for discussion purposes) for the bubble surface area is given as:

$$
\frac{\partial S_{g}}{\partial t}+\frac{\partial S_{g} u}{\partial x}=s_{t}
$$

Here $s_{t}$ is the source terms to the cloud surface area equation derived in a manner consistent with the Rayleigh Plesset equation and is defined later. For clarity, we repeat the vapor mass conservation, which was already included as part of the original multi-phase system (Eqn. (2)),

$$
\frac{\partial \rho_{g} \phi_{g}}{\partial t}+\frac{\partial \rho_{g} \phi_{g} u}{\partial x}=m_{t}
$$

The dependent variables $S_{g}$ and $\phi_{g}$ may be written as

$$
S_{g}=N * 4.0 * \pi * r^{2}, \quad \phi_{g}=N * 4.0 * \pi * r^{3} / 3.0
$$

where $\mathrm{N}$ is the number density of bubbles, and $\mathrm{r}$ the Sauter mean radius of bubbles at each grid point. Thus, the Sauter mean radius, $\mathrm{r}$, that conserves cloud surface area and volume is defined as, $r=3 * \phi_{g} / S_{g}$. Note that no restriction is placed on the vapor volume fraction being dilute. Furthermore we do not restrict the number density of bubbles to be a constant in each cell, and allow them to convect as governed by the hydrodynamics.

The source terms for the net vapor mass transfer and surface area change are given by

$$
\begin{aligned}
& m_{t}=\rho_{g} S_{g} r_{t} \\
& s_{t}=2.0 * S_{g} r_{t} / r
\end{aligned}
$$

where $r_{t}$ is the rate of change of the radius. To specify the rate of change of radius $r_{t}$, the Rayleigh-Plesset Equation could be specified as follows:

$$
r \frac{d^{2} r}{d t^{2}}+\frac{3}{2}\left(\frac{d r}{d t}\right)^{2}=\frac{1}{\rho_{l}}\left\{\left(P_{i}-P_{\infty}\right)-\frac{2 \sigma}{r}-\left(\frac{4 \mu}{r}\right) \frac{d r}{d t}\right\}
$$

However this would require the solution of an additional transport equation for the solution of (dr/dt). In our study a simplified and approximate form for the radius change is used:

$$
\frac{d r}{d t}= \pm\left(\frac{2}{3}\right)^{1 / 2}\left(\frac{a b s\left(P_{v}-P\right)}{\rho_{l}}\right)^{1 / 2}
$$

where the sign of the radius change term depends on whether the bubble is growing or decaying and is dictated by the sign of the $P_{v}-P$ term. Here, $P_{v}$ is the vapor pressure, while $P$ is the instantaneous pressure at the grid node point at which the cavitation source term is being computed, and $\rho_{l}$ is the liquid density. We again emphasize that Eqn. (11) provides the phase change for an individual bubble only while the net mass transfer is given by Eqn. (9) by accounting for the total surface area of bubbles present at a given spatial grid location. Hence a more fundamental unsteady cavitation formulation is derived. 


\section{LES Models}

Unsteady simulations presented in this paper have been computed with a one-equation LES model unless otherwise noted. The one equation LES model solves for a transport equation for the sub-grid kinetic energy. This is then used to evaluate the eddy viscosity that is used to model the unresolved SGS stresses. The model equations are as follows

$$
\frac{\partial \bar{\rho} \tilde{k}}{\partial t}+\frac{\partial}{\partial x_{i}}\left(\bar{\rho} \tilde{u}_{i} \tilde{k}-\bar{\rho} v_{T} \frac{\partial \tilde{k}}{\partial x_{i}}\right)=\tau_{i j}^{s g s} \frac{\partial \tilde{u}_{i}}{\partial x_{j}}-D_{k}
$$

where, $\tilde{k}$ is the Favre filtered subgrid kinetic energy, $\tilde{u}_{i}$ is the resolved scale velocity, $\bar{\rho}$ is the filtered density, $\tau_{i j}^{s g s}=\bar{\rho}\left(u_{i} u_{j}-\tilde{u}_{i} \tilde{u}_{j}\right)$ is the subgrid scale tensor, $v_{T}$ is the subgrid scale eddy viscosity and $D_{k}$ is the dissipation of the SGS kinetic energy. The subgrid scale stresses are modeled as

$$
\tau_{i j}^{s g s}=-2 \bar{\rho} v_{T}\left(\tilde{S}_{i j}-\frac{1}{3} \delta_{i j} \tilde{S}_{k k}\right)+\frac{2}{3} \bar{\rho} \tilde{k} \delta_{i j}
$$

The eddy viscosity is computed as

$$
v_{T}=C_{v} \tilde{k}^{1 / 2} \Delta
$$

where, $C_{v}$ is the modeling constant and has been computed to be equal to 0.0667 (Arunajatesan ${ }^{10}$ ).

\section{Validation for Unsteady Test Cases}

Rigorous validation of the cavitation model has been performed for unsteady simulations. The following test cases are presented here: 1) Bubble dynamics in a fluctuating pressure environment for validating the cloud surface area equations; 2) Unsteady cloud cavitation on an ogive in liquid nitrogen.

\section{A. Bubble Dynamics in a Fluctuating Pressure Environment}

Unsteady, one-dimensional, bubbly pipe flow is analyzed; the stagnation pressure at the inlet is constant while the back pressure fluctuates periodically. For dilute bubble concentrations, an analytical solution is derived for the pressure and velocity of the fluid. The bubble response at various forcing frequencies is obtained by injecting bubbles at the inflow and tracking their growth/decay using a Lagrangian procedure where the pressure and velocity at each instant is specified using the analytical solution; this is referred to as "exact" bubble solution. The numerical solution is obtained using the Eulerian CFD framework. Both the mean liquid solution and the bubble dynamics are computed. Thus rigorous validation of bubble dynamics is achieved with spatial and temporal variations of the mean flow.

The analytical solution for the velocity and pressure fluctuations is derived to be (Athavale ${ }^{11}$ ),

$$
\begin{aligned}
& u^{\prime}(t)=-\left(\frac{\varepsilon}{\rho_{L} u_{0}^{2}}\right)\left(\frac{u_{0}}{1+\Omega^{2}}\right)\left[\sin w t-\Omega \cos w t+\Omega e^{\frac{-u_{o} t}{L}}\right], \\
& P^{\prime}(x, t)=\left[\varepsilon \sin w t+\rho u_{0} u^{\prime}\right] \frac{x}{L}-\rho u_{0} u^{\prime}, \ldots \Omega=\frac{w L}{u_{0}}, \\
& \text { Phase lag } \phi=\tan ^{-1}(\Omega)
\end{aligned}
$$

where, $\Omega$ is the non-dimensional frequency, and $\varepsilon$ is the amplitude of the pressure fluctuations enforced at the exit. Note that the velocity fluctuations are uniform in space, while the pressure fluctuations show a linear decay from the exit to the inflow as a function of the frequency of forcing.

The temporal history of the solution is shown in Figure 1 for three different non-dimensional frequencies of $\Omega=1,10$, and 100 . In all these cases, the amplitude of the pressure fluctuations at the exit was kept at ten percent of the mean pressure. Figures $1 \mathrm{a}$ and $1 \mathrm{~b}$ show the pressure fluctuation at the inlet and exit, while Figure $1 \mathrm{c}$ shows the corresponding bubble history at the exit. Excellent comparisons are obtained overall. At the lowest frequency $(\Omega=1)$, the pressure drop between the inlet and exit is the lowest. As the frequency increases, the pressure drop 
increases significantly and by $\Omega=100$, the pressure fluctuations at the inflow are almost negligible. The temporal history of fluctuations in bubbles radius at the exit of the pipe shows a similar behavior; with the bubble growth being most responsive at the lower frequencies. Indeed at the highest frequency $(\Omega=100)$, the bubble convects with negligible change in radius even though the amplitude of the local pressure fluctuation at the exit is unchanged. We caution that these results were obtained for pure vapor bubbles. For non-condensable gas bubbles, the physics is far more complicated since the bubbles have a natural frequency and may show resonant behavior.

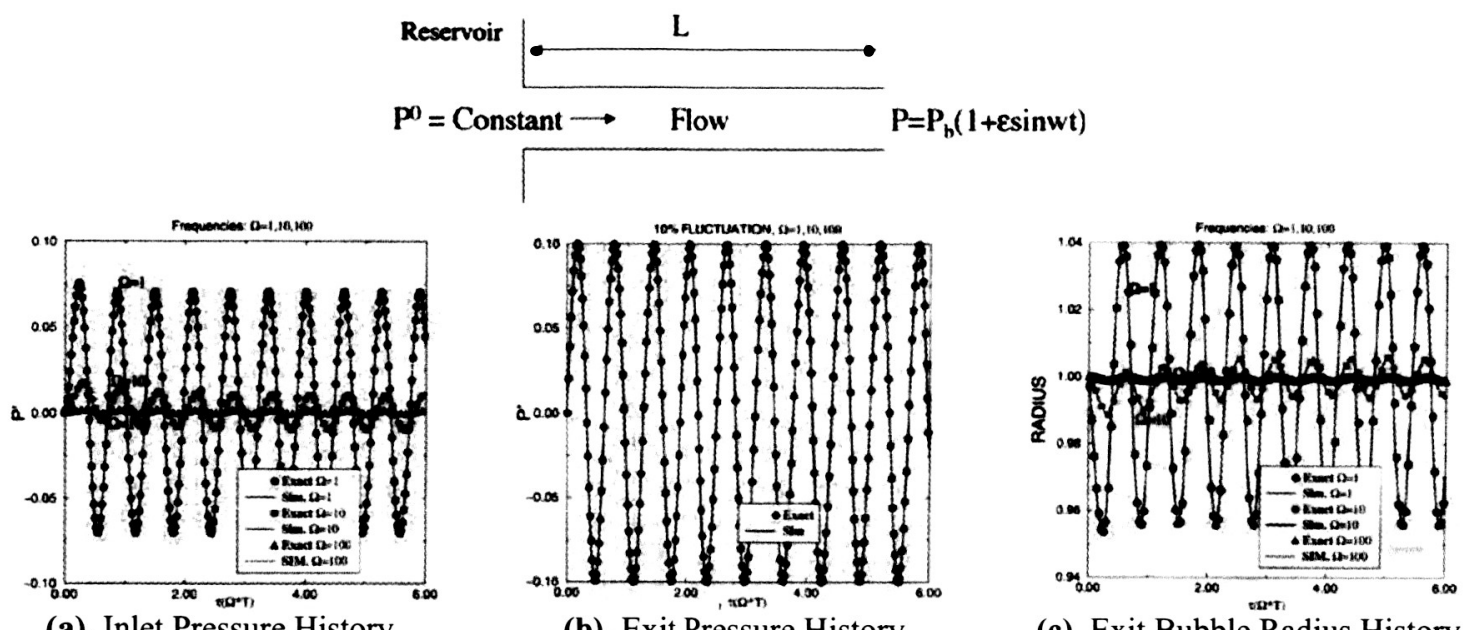

(a). Inlet Pressure History.

(b). Exit Pressure History.

(c). Exit Bubble Radius History.

Figure 1. Flow Solution History For Bubbly Unsteady Pipe Flow, $\Omega=1,10,100$.

\section{B. Cavitating Ogive Simulations}

We perform unsteady simulations of experiments by Hord ${ }^{12}$ on a cavitating ogive to validate the formulation for cavitation in cryogenic fluids. Hord performed sub-scale tests using both liquid nitrogen and hydrogen in a blowdown tunnel. The details of the tunnel and the ogive geometry are given in Figure 2. The tunnel width is 1.158 inches while the ogive diameter is 0.357 inches. Hence considerable blockage effects from the tunnel wall are present and the simulation modeled the tunnel geometry. To ensure that the tunnel blockage effects were being correctly modeled we performed single-phase, non-cavitating simulations and have compared it to Hord's noncavitating data in Figure 3. Good agreement is obtained giving confidence that the tunnel interaction is being captured.

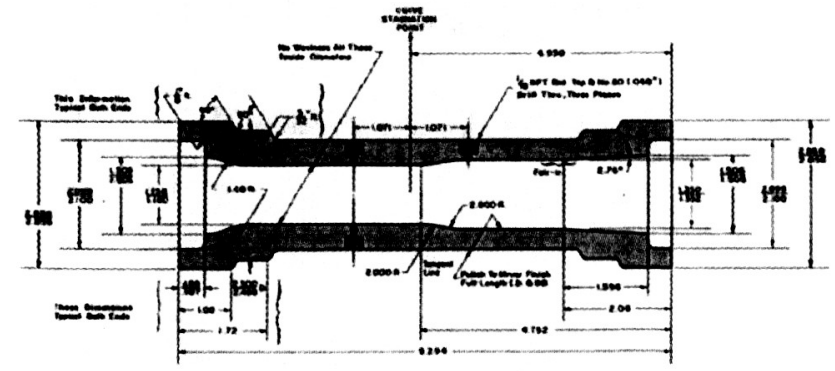

(a)Tunnel configuration

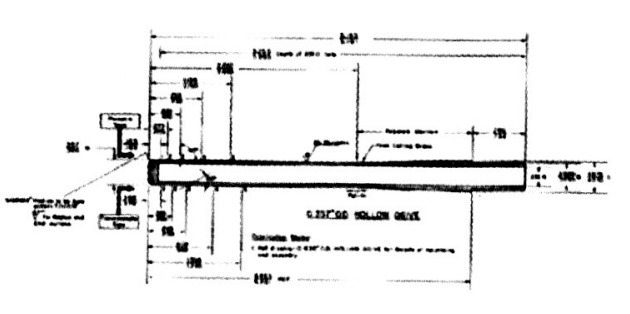

(b)Ogive test article

Figure 2. Details of Subscale Tunnel and Ogive Test Article From Hord (1973 (b)).

Simulations are performed at the following flow conditions in liquid nitrogen: inlet temperature of $83 \mathrm{~K}$, inlet velocity of $23.5 \mathrm{~m} / \mathrm{s}$, inlet pressure of $2.87 \mathrm{~atm}$, with a freestream vapor pressure of $1.875 \mathrm{~atm}$. Unsteady calculations for the following three different viscosity models are compared: 1) RANS $k-\varepsilon$ model, 2) one equation LES model, and 3) no sub-grid viscosity (or laminar) model. Fig. 4 (a-d) shows the flowfield contours for the unsteady calculation computed with the RANS model. As is apparent from these figures the flow remains essentially steady despite the calculation being performed in an unsteady mode; the vapor volume fraction and

5

American Institute of Aeronautics and Astronautics 
temperature (Fig. $4 \mathrm{a}$ and $4 \mathrm{c}$ ) show a uniform variation with the largest temperature depression occurring at the leading edge. The steady flowfield results from large turbulent viscosities in the cavity closure region (Fig. 4d) that damps the strength of the reentrant jet which as we shall discuss subsequently plays a key role in driving the cavity unsteady. The comparisons of pressure and temperature depression are compared with experimental data in Fig. 5. The experimental temperature values did not recover to the freestream temperature of $83^{\circ} \mathrm{K}$ and instead asymptoted to a value of $82.7^{\circ} \mathrm{K}$. It was unclear if this difference was due to error in measurement of either the freestream or the ogive temperature, or whether this was caused by heat transfer losses. These values were shifted by $0.3^{\circ} \mathrm{K}$ to match the stated freestream value of $83^{\circ} \mathrm{K}$. We observe that the results that are consistent with steady calculations; the leading edge temperature and pressure depression compare well with data but the cavity length is smaller as noted by the more abrupt temperature and pressure recovery.

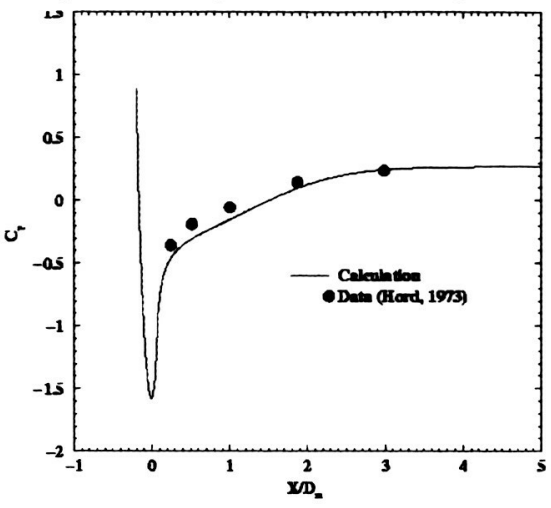

Figure 3. Pressure Coefficient for Single Phase Flow in Sub-Scale Tunnel.

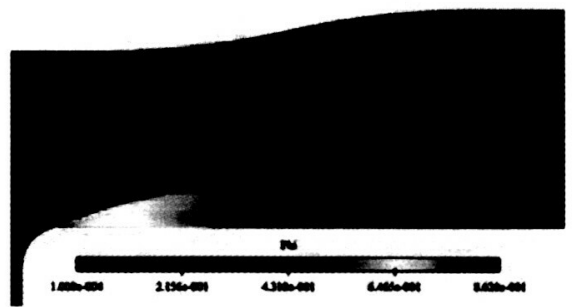

(a) Vapor volume fraction

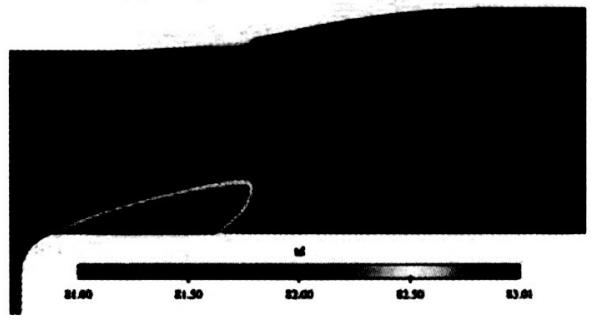

(c) Temperature

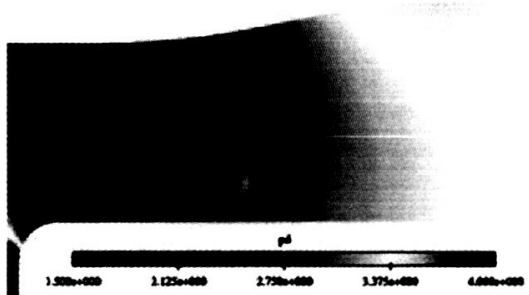

(b) Pressure

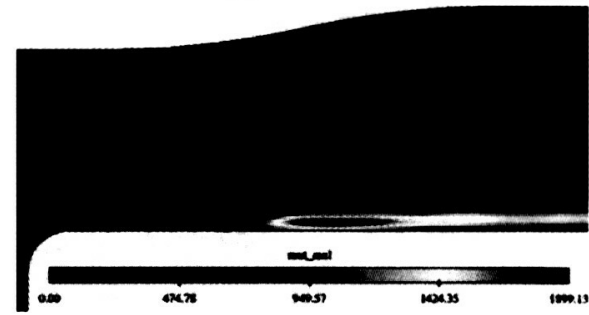

(d) Turbulent viscosity

Figure 4. Flowfield Contours for Cavitating Ogive Using a RANS Turbulence Model.
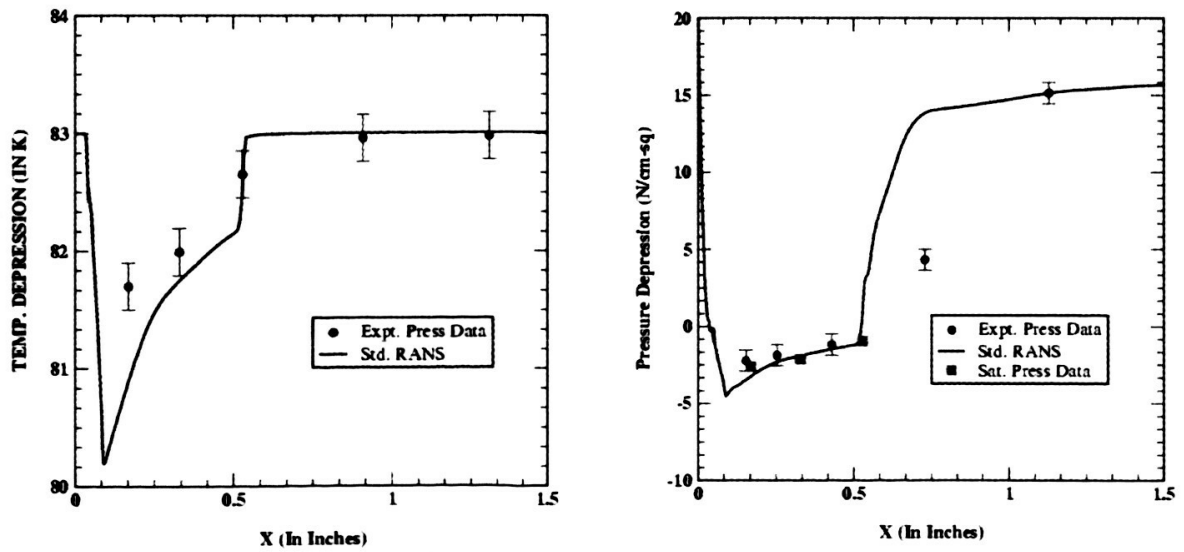

Figure 5. Comparison of Pressure and Temperature Depression completed with RANS Turbulence Model with Data.

6

American Institute of Aeronautics and Astronautics 


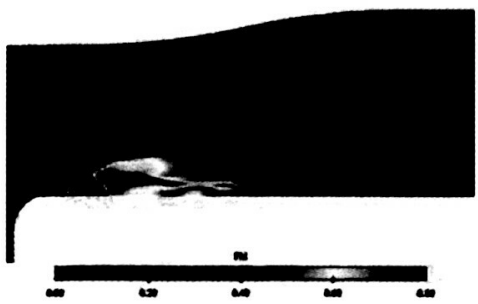

(a)

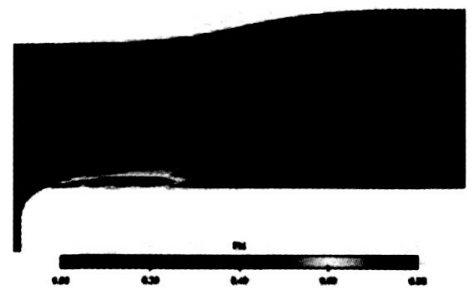

(d)

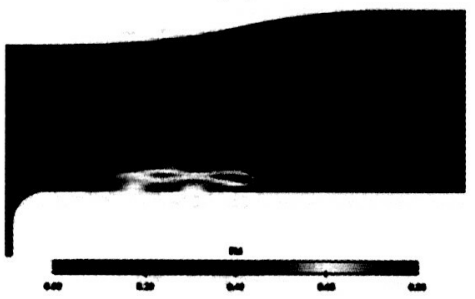

(g)

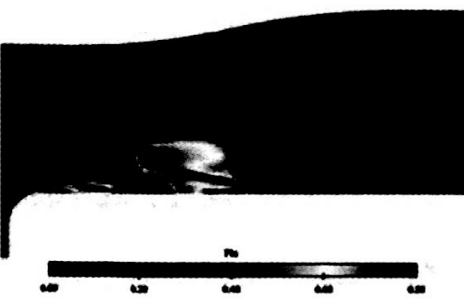

(b)

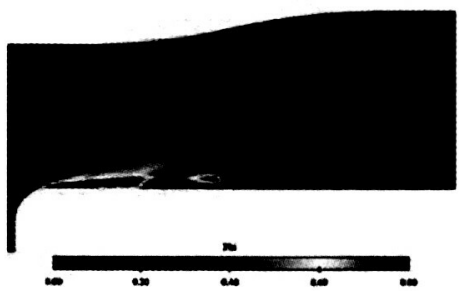

(e)

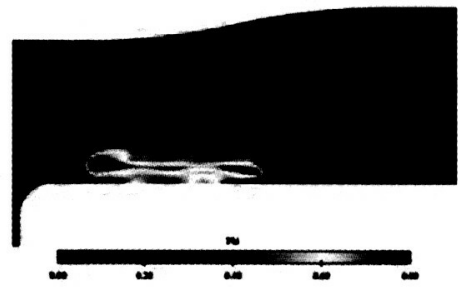

(h)

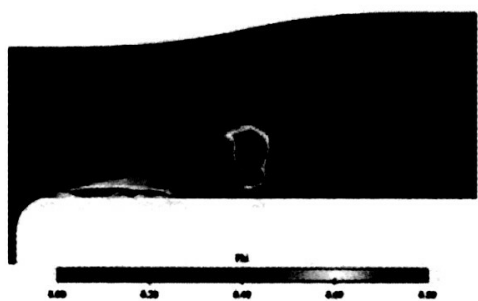

(c)

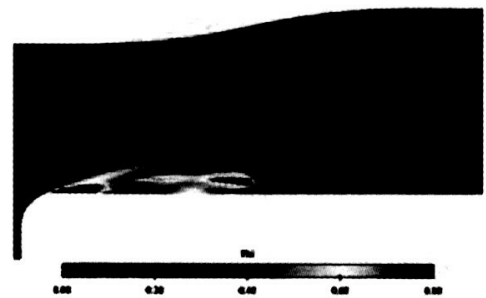

(f)

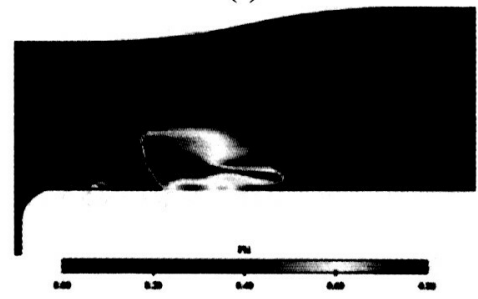

(i)

Figure 6. Instantaneous Vapor Volume Fraction Contours for Unsteady LES Calculations Shown at Intervals of $0.29 \mathrm{~ms}$.

Simulations at the same conditions but computed with the one-equation LES model rather than a RANS turbulence model generated large scale flow unsteadiness. Figure 6 shows snapshots of vapor volume fraction at ten snapshots which are $0.29 \mathrm{~ms}$ apart (or roughly $1 / 10^{\text {th }}$ of a $340 \mathrm{~Hz}$ frequency). A periodic shedding is observed where the main cavity appears to get sheared and lifted-off the leading edge before being convected downstream. Figure 7 shows the vapor volume fraction contours overlaid with the velocity field. It indicates that the shearing action arises due to fluid flowing back in a narrow region near the ogive surface. Thus the interaction of the reentrant jet with the boundary layer of the oncoming flow appears to play a critical role in determining the unsteady characteristics of the cavity.

The instantaneous temperature and pressure contours are shown in Figures 8 and 9 respectively. One consequence of the unsteady shedding is that the minimum temperature is not necessarily at the leading edge but at the core of the traveling vapor cloud as it convects downstream. In fact the temperature at the leading edge location rises sharply each time the cavity gets sheared off and this will be reflected in the mean temperature profiles that we discuss later. The pressure contours reflect the highly dynamic flowfield where local pressure excursions are observed as condensation occurs in the region between vapor clouds. A detailed analysis of the frequency content and amplitudes of the pressure fluctuations will be provided later in this section.

The time-averaged mean solution of the instantaneous flowfields taken over a large time sample are plotted in Fig. 10(a-c). Despite the large scale unsteadiness exhibited by the flow, the mean solutions look qualitatively similar to the steady solutions obtained with the RANS turbulence model. However some significant differences are also apparent. The cavity length is much longer and the variation of temperature and volume fraction much less in the LES mean solutions. A comparison of the temperature and pressure depression is compared to the experimental data as well as the RANS solution in Fig. 11. We note that both the temperature and pressure profile in the cavity closure region for the LES calculation show a more gradual recovery similar to the data and this is clearly arising from the unsteady effects which result in lower temperature and pressure vapor clouds convecting downstream in a periodic fashion. At the leading edge however, the temperature recovers more rapidly and is higher than the data over most of the cavity. This may also be attributed to unsteady effects since the leading edge of the cavity is not attached and experiences temperature increases due to condensation. 


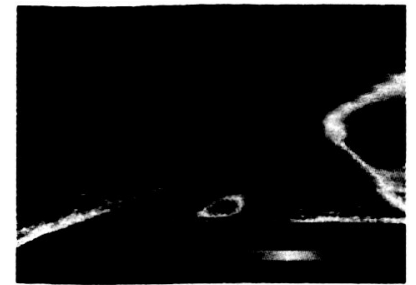

(a)

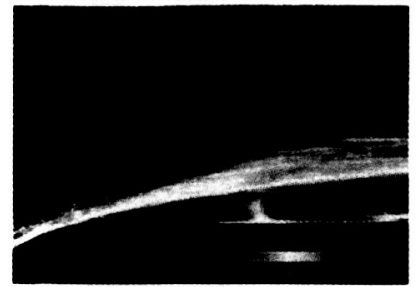

(d)

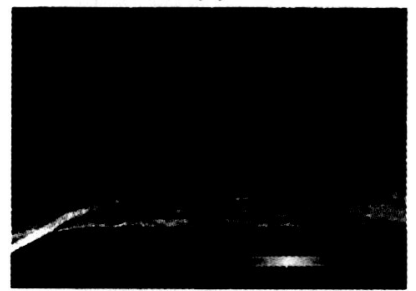

(g)

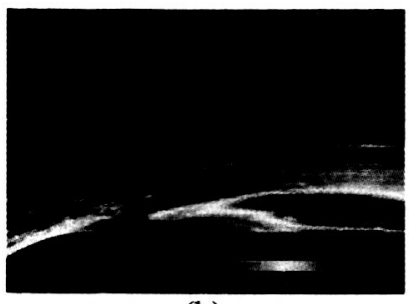

(b)

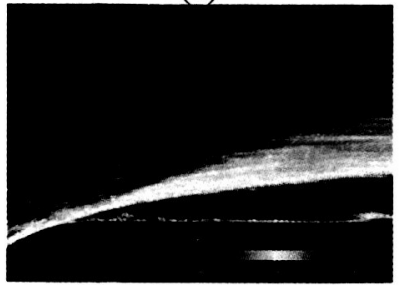

(e)

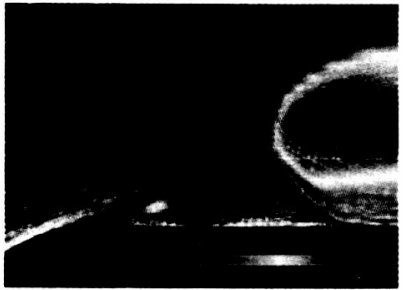

(h)

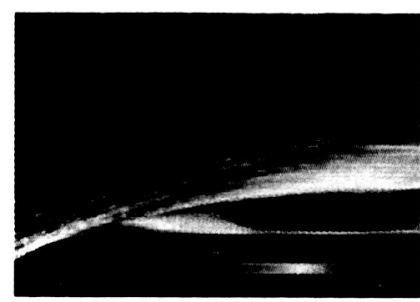

(c)

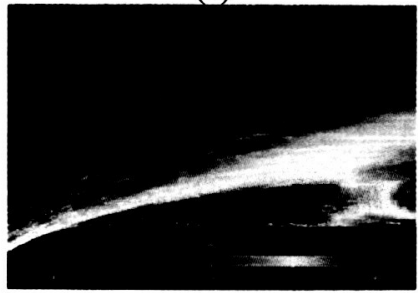

(f)

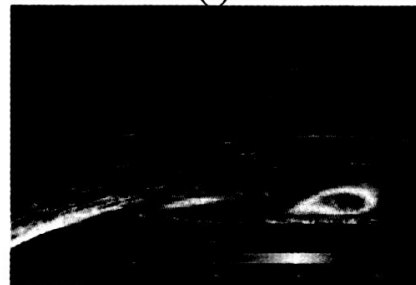

(i)

Figure 7. Instantaneous Velocity Vector Contours for Unsteady LES Calculation Shown at Intervals of 0.29ms.

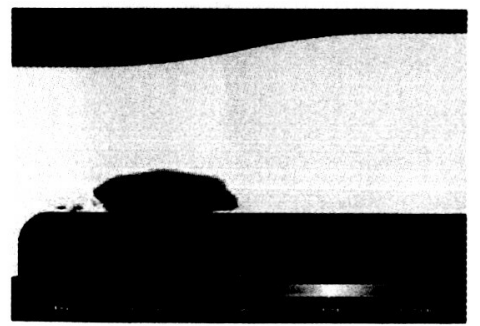

(a)

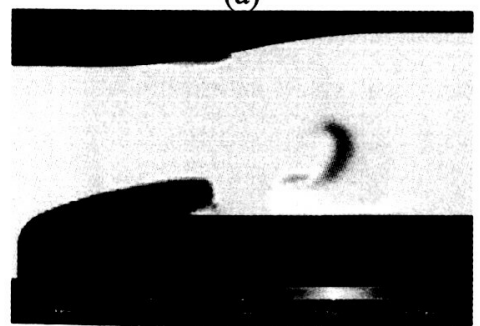

(d)

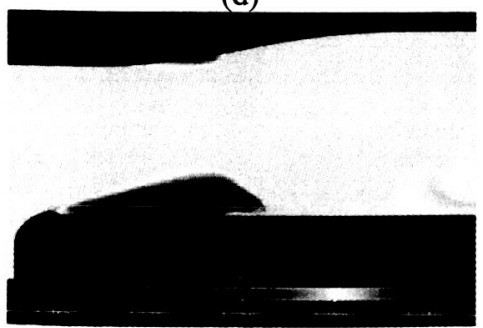

(g)

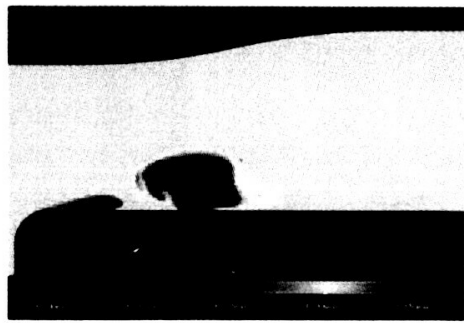

(b)

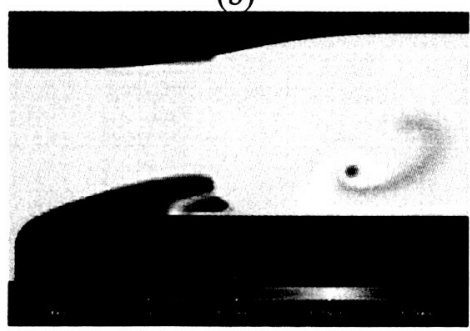

(e)

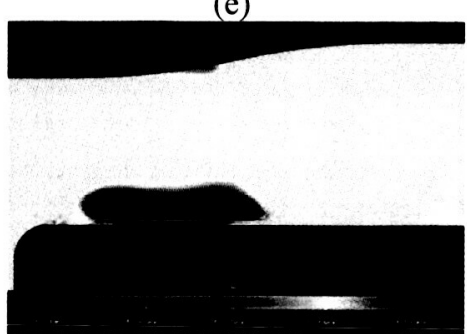

(h)

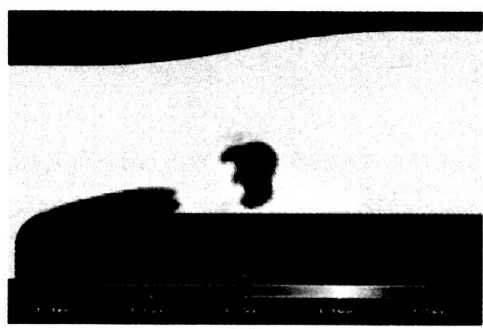

(c)

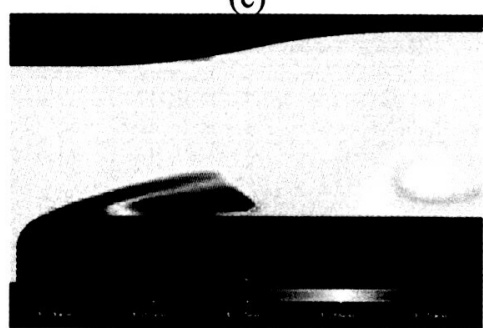

(f)

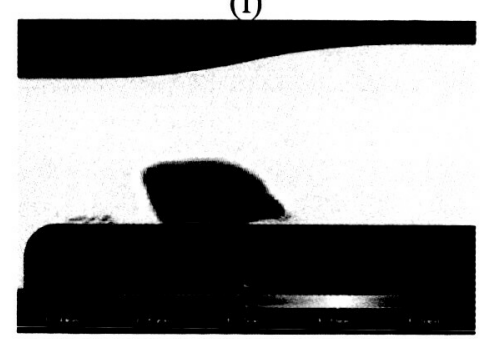

(i)

Figure 8. Instantaneous Temperature Contours for Unsteady LES Calculation Shown at Intervals of 0.29ms. 


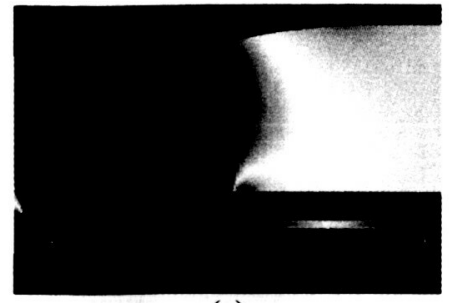

(a)

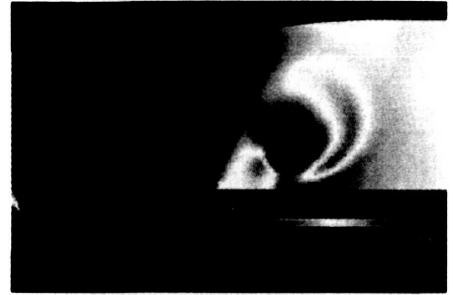

(d)

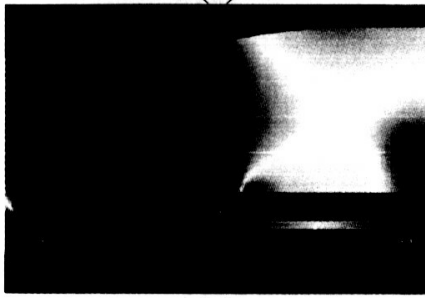

(g)

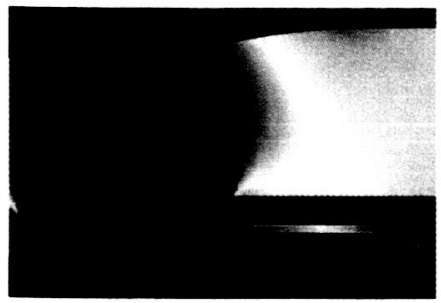

(b)

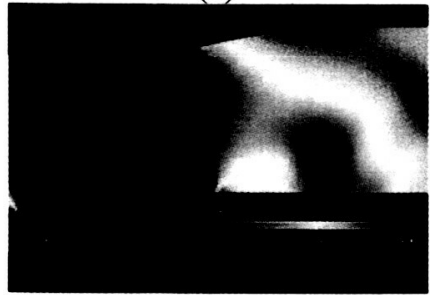

(e)

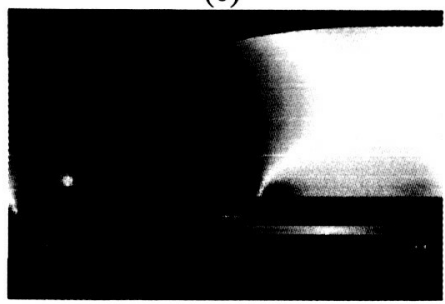

(h)

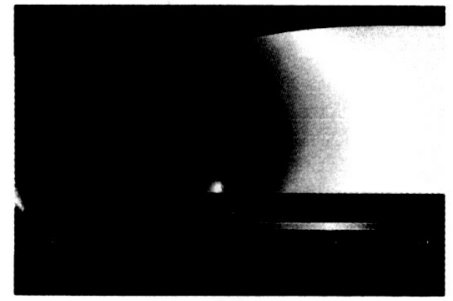

(c)

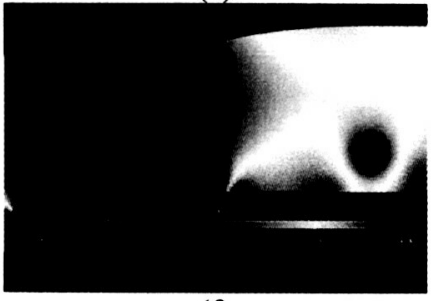

(f)

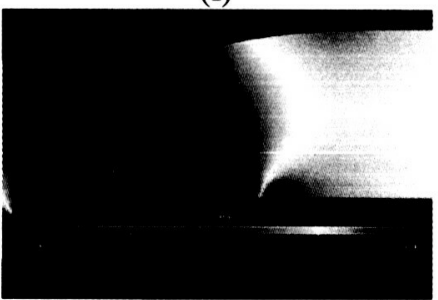

(i)

Figure 9. Instantaneous Pressure Contours for Unsteady LES Calculation Shown at Intervals of $0.29 \mathrm{~ms}$.

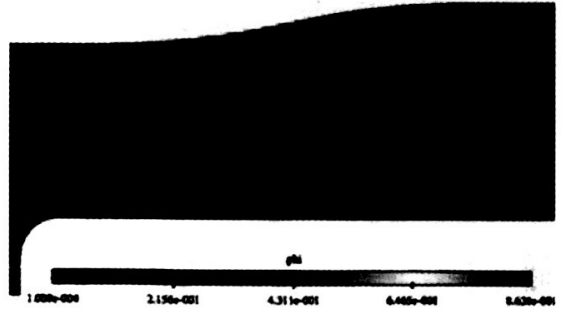

(a) Vapor volume fraction

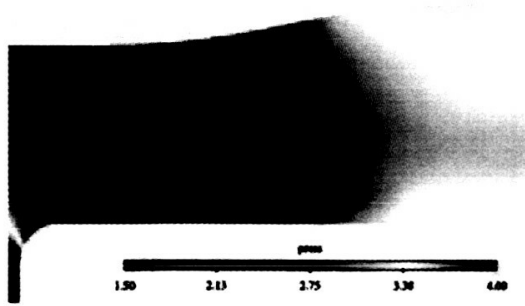

(b) Pressure

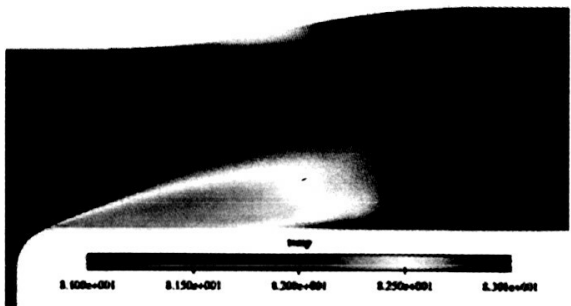

(c). Temperature

Figure 10. Time Averaged Mean Flowfield Contours from Unsteady LES Calculation.
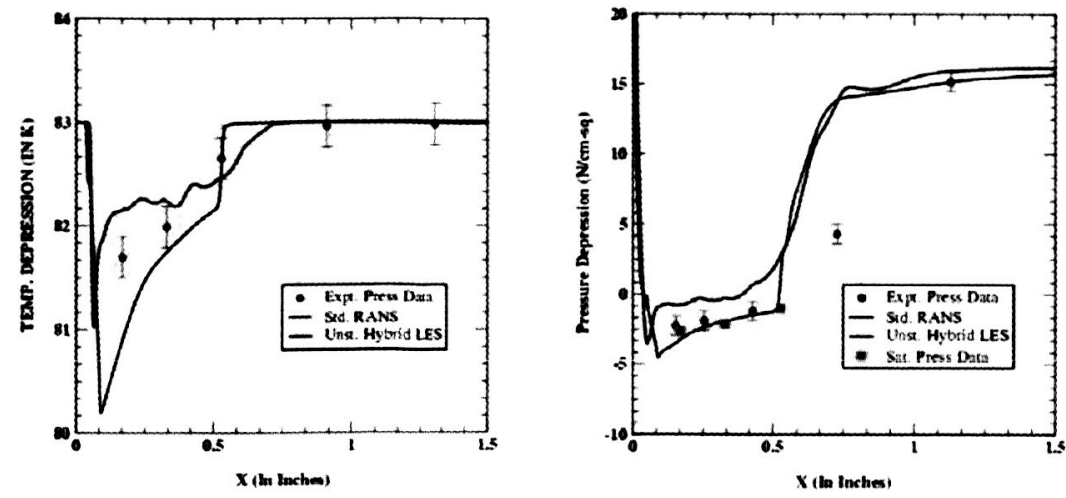

Figure 11. Comparison of Mean Pressure and Temperature Depression from Unsteady LES Calculations with RANS Solution and Experimental Data.

9

American Institute of Aeronautics and Astronautics 
To further illustrate the strong sensitivity of the solution to the interactions between the reentrant jet and the boundary layer, we compute a third solution with no sub-grid viscosity where the boundary layer is laminar. Figure 12 compares the time-averaged mean temperature and pressure depression profiles for the LES calculation with unsteady laminar calculation. We note that the reduced viscous damping amplifies the differences we observed in our earlier comparison of the RANS and LES solutions. Specifically, the laminar solution (brown line in Fig. 13) shows an even larger mean cavity length with a slower pressure and temperature recovery and compares well with data in the closure region. However, the temperature and pressure rise at the leading edge is quicker and the comparison with experimental data gets worse here.

The dynamic flow fluctuations arising from the unsteady cavitation physics are examined by plotting the pressure, temperature and volume fraction fluctuations close to the leading edge of the cavity in Fig. 13. The

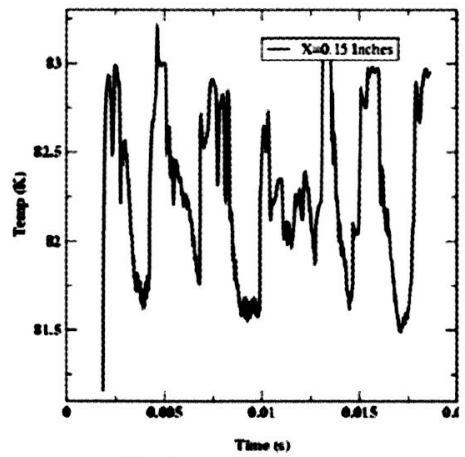

(a) Temperature

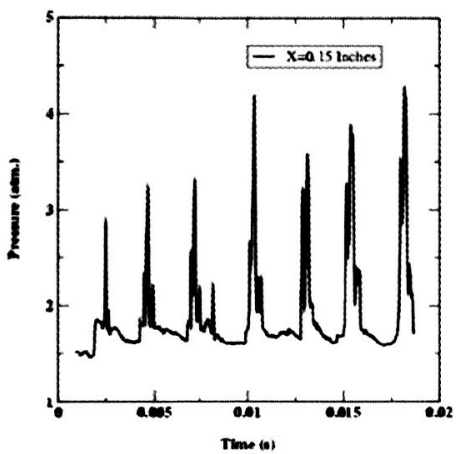

(b) Pressure

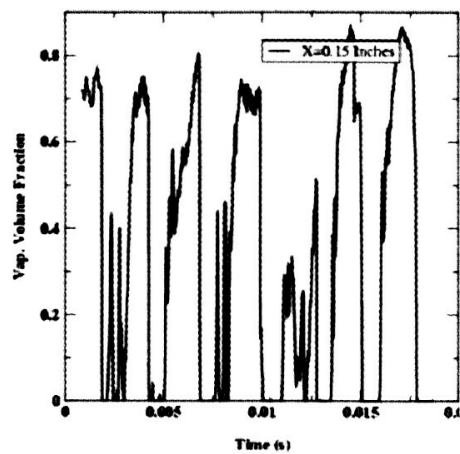

(c) Volume fraction

Figure 13. Time Traces of Flow Variables Close to the Leadings Edge of Cavity on Ogive.

pressure fluctuations show periodic large amplitude pressure spiking where the amplitude of the peak pressure excursion is approximately $100-200 \%$ of the mean pressure. Although the frequency between the peaks corresponds to a lower frequency associated with the cavity shedding, the pressure excursion itself occurs over a very small time scale. Spectral analysis of these pressure fluctuations shown in Fig. 14 indicates that the bulk of the energy is in the shedding frequency mode of $400 \mathrm{~Hz}$ with a rapid drop-off in energy amplitudes at the higher overtones at $1 \mathrm{KHz}$ and beyond. The rms value of the fluctuations is $0.49 \mathrm{~atm}$ for a mean pressure level of $1.89 \mathrm{~atm}$ (i.e. $25.9 \%$ fluctuation level) which indicates a substantial dynamic load that can potentially cause damage locally.

The temperature and volume fraction time history plotted in Fig. 13 indicate that when large scale instabilities occur the temperature fluctuations are of the order of the mean temperature depression itself and are driven by the hydrodynamic interactions between the vapor cloud and the

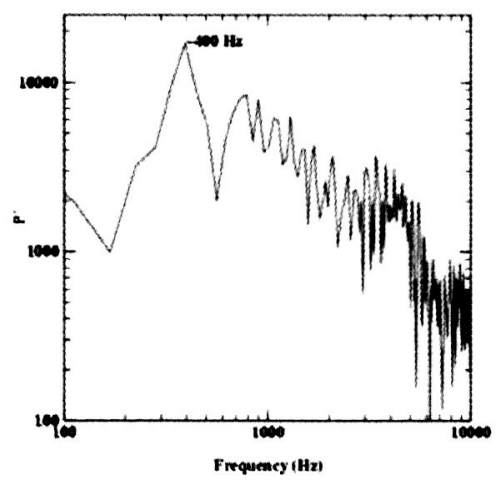

Figure 14. Frequency Spectrum of Pressure Oscillations Near the Leading Edge of Cavity.

pressure field. Consequently, the temperature fluctuations appear to be in-phase with the pressure fluctuations. We

10

American Institute of Aeronautics and Astronautics 
note that the large amplitude of the temperature fluctuations results in the average temperature rising and this was evident on the mean temperature profiles compared in Fig. 12. The volume fraction time history confirms that shedding of the vapor clouds results in periods when the ogive is "wetted" by liquid nitrogen even at a location close to the leading edge of the cavity.

\section{Conclusion}

An unsteady cavitation model for dense cloud cavitation that can predict amplitudes and frequencies of dynamic pressure loads, and operate within a generalized compressible "real" fluid framework, has been demonstrated. A fundamental formulation for the unsteady phase change that accounts for the bubble size and number density as well its coupling to the acoustic propagation in the multi-phase fluid mixture has been developed. Simulations of a cavitating ogive in liquid nitrogen indicate that unsteady shedding of cavities appears to be controlled by the interaction of the reentrant jet with the vapor cavity. Highly dynamic flowfields with large dynamic pressure fluctuations were observed with the appropriate treatment of the turbulence terms. The validity of the unsteady solutions was verified by time-averaging the solution and comparing mean profiles to experimental data; the comparison in the cavity closure regions improved significantly within an unsteady framework i.e. a combination of the new unsteady cavitation model and the LES turbulence model. However, we note that resolving unsteady interactions and separation for boundary layers in very high Reynolds number flows is extremely difficult; in particular the leading edge of the cavity may be more steady/stable than indicated by the LES calculations here and may require the use of a hybrid LES-RANS model.

\section{Acknowledgments}

We acknowledge funding for this work through a SBIR program under Contract No. NNM05AA05C funded by NASA Marshall Space Flight Center. The contract monitor is Dr. Dan Dorney. The technical inputs provided by Dr. Dan Dorney and Mr. Robert Garcia are gratefully acknowledged.

\section{References}

1 Kubota, A., Kato, H. and Yamaguchi, H., (1992) "Cavity Flow Predictions Based on the Euler Equations," J. Fluid Mech., Vol. 240, pp. 59-96.

2 Colonius, T., d'Auria, F., Brennen, (2000) C.E., "Acoustic Saturation in Bubbly Cavitating Flow Adjacent to an Oscillating Wall," Phys. Fluids, Vol.12, No.11, pp. 2752-2761.

3 Song, C.S.S., and Qin, Q., (2001) "Numerical Simulations of Unsteady Cavitating Flows", Proceedings of the Fourth International Symposium on Cavitation, Pasadena.

4 Merkle, C.L., Feng, J.Z. and Buelow, P.E.O., (1998) "Computational Modeling of the Dynamics of Sheet Cavitation," Proceedings of the 3rd International Symposium on Cavitation, Grenoble.

5 Singhal, A.K., Athavale, M.M., Li, H., and Jiang, Y., (2002) "Mathematical Basis and Validation of the Full Cavitation Model," J. Fluids Engg., Vol.124 (No.3), pp. 617-624.

6 Ahuja, V., Hosangadi, A. and Arunajatesan, S., "Simulations of Cavitating Flows Using Hybrid Unstructured Meshes," Journal of Fluids Engineering, June 2001.

7 Hosangadi, A. and Ahuja, V., "A Numerical Study Of Cavitation In Cryogenic Fluids," Journal of Fluids Engg., To be Published May 2005.

${ }^{8}$ Lemmon, E.W., Peskin, A.P., McLinden, M.O., and Friend, D.G., (2000) "NIST Thermodynamic and Transport Properties of Pure Fluids", NIST Standard Reference Database 12, Users Guide.

9 Hosangadi, A., Sinha, N., and Dash, S.M., "A Unified Hyperbolic Interface Capturing Scheme for Gas/Liquid Flows," AIAA-97-2081, 13th AIAA CFD Conferences, Snowmass, CO, June 29-July 2, 1997.

${ }^{10}$ Arunajatesan, S., Sinha, N., and Menon, S., "Towards Hybrid LES-RANS Computations of Cavity Flowfields," AIAA Paper No. 2000-0401, 38 ${ }^{\text {th }}$ AIAA Aerospace Sciences Meeting at Reno, NV, Jan 10-13, 2000.

${ }^{11}$ Athavale, M.M., and Singhal, A.K., "Numerical Analysis of Cavitating Flows in Rocket Turbopump Elements", $37^{\text {th }}$ AIAA Joint Propulsion Conference, July 2001.

${ }^{12}$ Hord, J., (1973) "Cavitation in Liquid Cryogens III -- OGIVES," NASA CR-2292. 